\title{
La contribution bolivienne à la COP 21 au prisme de la justice environnementale
}

Florence Bétrisey and Christophe Mager

\section{(2) OpenEdition}

\section{Journals}

Electronic version

URL: http://journals.openedition.org/echogeo/14442

DOI: $10.4000 /$ echogeo.14442

ISSN: 1963-1197

\section{Publisher}

Pôle de recherche pour l'organisation et la diffusion de l'information géographique (CNRS UMR 8586)

Electronic reference

Florence Bétrisey and Christophe Mager, « La contribution bolivienne à la COP 21 au prisme de la justice environnementale», EchoGéo [Online], Sur le Vif, Online since 27 January 2016, connection on 03 May 2019. URL : http://journals.openedition.org/echogeo/14442 ; DOI : 10.4000/echogeo.14442

This text was automatically generated on 3 May 2019.

\section{(c) (i) (9)}

EchoGéo est mis à disposition selon les termes de la licence Creative Commons Attribution - Pas d'Utilisation Commerciale - Pas de Modification 4.0 International 


\section{La contribution bolivienne à la COP 21 au prisme de la justice environnementale}

Florence Bétrisey and Christophe Mager

\section{Introduction}

1 «La contribution nationale de la Bolivie en vue de la COP 21 classe le changement climatique dans les échecs de l'économie capitaliste, auquel le pays appelle à mettre fin » (Barbière, 2015, s.p.), pouvait-on lire le 13 octobre 2015 sur le site d'information en ligne EurActiv.fr. La délégation bolivienne aux Nations Unies entendait en effet, dans sa « contribution (...) déterminée au niveau national $»^{1}$ pour la COP 21 , contester et bannir le "modèle de civilisation consumériste, belliciste et mercantiliste du capitalisme» (Ministerio de Planificación del Desarrollo, 2015). La remise en question du modèle actuel de distribution de droits à émettre des gaz à effets de serre (GES) entre les États était le levier principal sur lequel la Bolivie se proposait d'agir afin d'œuvrer pour une gouvernance climatique globale plus «juste » (Ministerio de Planificación del Desarrollo, 2015).

2 Sachant que le caractère pluriel et polysémique de la justice est aujourd'hui largement reconnu (Sikor et al., 2014), le discours et les solutions proposées par le gouvernement bolivien amènent à s'interroger, dans un premier temps, sur les conceptions de la justice sur lesquelles se fonde la contribution bolivienne. Pour y répondre, nous reviendrons brièvement sur les différentes interprétations de la justice au sein du courant de la justice environnementale dans lequel s'insère la notion de justice climatique évoquée dans la contribution bolivienne. Nous ferons résonner cette dernière avec des fragments de discours recueillis par les auteurs depuis 2012 lors de participations à des conférences publiques données par des membres de la délégation bolivienne aux Nations Unies. Les discours du Président Evo Morales et de membres de son gouvernement seront aussi 
utilisés, tels que publiés dans des médias (télévisions et journaux) (non-)boliviens entre 2012 et 2015.

3 Dans un deuxième temps, nous montrerons comment, alors que le gouvernement bolivien se fait, discursivement, le chantre d'une promesse de renversement du modèle eurocentriste -pour reprendre l'expression d'Amin (1988)-, ses propositions ont paradoxalement pour incidence une légitimation, au moins partielle, du recours à un modèle de développement extractiviste au service d'une croissance chrématistique ${ }^{2}$ (Martínez-Alier et al., 2010).

\section{La multi-dimensionnalité de la notion de justice appliquée à l'environnement}

4 La notion de justice climatique évoquée dans les diverses stratégies se proposant de lutter contre le réchauffement climatique se réfère usuellement à une utilisation juste de l'espace atmosphérique, considéré comme une ressource environnementale commune (Page, 2011). L'annexe de l'Accord de Paris ${ }^{3}$ à l'issue de la COP 21 y fait explicitement référence, arguant, selon Laurent Fabius, que les pays du Nord et ceux du Sud ont des «responsabilités communes mais différenciées » dans le changement climatique, et que leurs « capacités respectives » à y faire face sont inégales (cité dans Hir, 2015, s.p.). La justice climatique peut être comprise comme une sous-catégorie de la justice environnementale, définie comme «la justice entre les humains, au sujet de questions environnementales » (Schlosberg, 2007, vii, trad. pers.). Il s'agit donc bel et bien d'une justice n'ayant pas comme objectif premier la protection de la nature, mais l'équité entre les individus, ce qui encourage certains auteurs à la considérer comme une "souscatégorie de la justice sociale » (Vanderheiden \& Sisson, 2010, p. 3, trad. pers.). Elle se place ainsi dans une vision anthropocentrée de la nature et se différencie de ce que Schlosberg (2007) appelle la justice écologique qu'il définit comme la justice envers la nature.

5 La notion de justice appliquée aux ressources et dommages environnementaux n'est pas récente, Blanchon et al. (2009) estimant que les mouvements sociaux des années 1980 aux États-Unis, « en relation étroite avec les mouvements des droits civiques» (op. cit., p. 37), sont les premiers à s'être revendiqués de la justice environnementale proprement dite. Sur le plan théorique, les travaux de Young (1990) et de Harvey (1996) sont considérés comme fondateurs du courant de justice environnementale. Sur cette base, les théories initiales de la justice environnementale, ainsi que les études empiriques s'y référant, se sont concentrées sur l'inégale répartition, parmi la population, des dysfonctionnements et dommages environnementaux (environmental burdens). Ce focus s'est par la suite élargi, en nourrissant largement la political ecology (Robbins, 2011), à l'étude de l'inégale répartition des bénéfices dérivés de l'environnement, ainsi qu'à l'inégale répartition des responsabilités et des coûts liés à la gestion des ressources naturelles (Walker, 2009). La dimension (re)distributive de la justice tient donc une place centrale dans ces théories initiales. 


\section{La justice environnementale comme juste redistribution}

6 L'idée d'une juste redistribution est issue d'une vision rawlsienne de la justice, fondée sur le principe de compensation des inégalités injustes (soit celles ne favorisant pas les plus démunis) et sur la reconnaissance d'inégalités justes (menant à la discrimination positive et aux initiatives pro-poor favorisant les plus moins bien dotés). La compréhension de la justice environnementale redistributive dépasse toutefois Rawls. Elle associe des références (1) à la question de l'accès aux ressources naturelles comme un droit fondamental (Perreault, 2014), ainsi (2) qu'au courant néo-marxiste, présent chez Harvey (1996), pour qui les inégalités environnementales sont « des formes d'oppression devant être traitées par une remise en cause du système capitaliste (...) [permettant d'aller vers] un système de production engendrant un juste partage des ressources naturelles " (Blanchon et al., 2009, p. 46). À titre d'exemple, Schroeder et al. (2008), dans leur introduction au numéro spécial de la revue Society \& Natural Resources: an International Journal consacré à la justice environnementale dans le Tiers-Monde, considéraient que les inégalités environnementales trouvaient leur origine dans « les décisions prises par des acteurs exogènes puissants, qui invoquèrent des récits basés sur le développement, la modernité et le progrès pour légitimer des gains privés » (op. cit., p. 553, trad. pers.). Ce courant donna naissance à une conception de la justice environnementale en tant que "structure ethno-environnementale » (Anthias \& Radcliffe, 2013, cité dans Joshi, 2015, p. 113, trad. pers.), les inégalités environnementales y étant vues comme des formes d'« impérialisme écologique » (Martin, 2013, p. 101, trad. pers.) ayant leur source dans la dépendance structurelle des pays en voie de développement envers les pays riches.

7 Bien que fortement liées à la justice sociale (que ce soit en tant que catégorie parallèle ou sous-catégorie), les théories de la justice environnementales ont peu pris en compte la pluralité des principes de justice au sein de la dimension distributive (lesquels définissent ce qu'est une distribution juste des dommages-bénéfices liés à l'environnement). D'autre part, les théories dites de " première génération » de la justice environnementale ont été myopes aux critiques de la justice distributive portant sur son caractère conséquentialiste, soit sur le fait qu'elle n'évalue moralement l'action qu'en aval (Policar, 2006). En d'autres termes, par exemple, l'évaluation est strictement centrée sur les conséquences ou les résultats de l'action (p.e. la justice de la redistribution) et néglige le caractère (in)juste des processus en amont aboutissant à tel ou tel modèle de distribution, que celui-ci soit considéré comme juste ou non. De même, ces théories de "première génération » n'ont que peu tenu compte des réflexions sur la multi-dimensionnalité de la notion de justice (et l'inclusion des dimensions de participation et de reconnaissance), bien que ces dernières aient toujours été présentes, certes parfois implicitement, au sein des revendications et des discours des mouvements de justice environnementale (Schlosberg, 2007). Enfin, bien que reconnaissant la nécessité de traiter les injustices environnementales ainsi que leurs déterminants institutionnels et politico-économiques, ces théories redistributives "macrosociologiques ou politico-économiques " (Flitner, 2009 , p. 400) ont eu tendance à rechercher des principes universels de justice " pouvant servir de références pour des jugements normatifs ultérieurs » (Martin et al., 2014, p. 168, trad. pers.). Partant, ces théories de la justice environnementale se sont vues reprocher leur « insuffisance et leur caractère inadéquat pour répondre aux tâches à la fois de révéler les inégalités de même que de comprendre les processus à travers lesquels ces dernières sont (re)produites » (Walker, 2009, p. 615, trad. pers.). 
8 C'est pourquoi les théories récentes de la justice environnementale, inspirées notamment par Sen (2002), se sont attachées à considérer la justice de manière plus pratique (Martin et al., 2014) et mieux fondée empiriquement. Ces approches de la justice environnementale ont ainsi mis en lumière sa dimension procédurale (e.g. dans le cadre des mécanismes de prise de décision relatifs à l'aménagement du territoire, aux politiques publiques environnementales, énergétiques, sanitaires ou encore agricoles). À la suite des travaux de Schlosberg $(2004,2007)$, en se basant sur les avancées théoriques de la justice sociale, elles se sont aussi attelées à démontrer la multi-dimensionnalité de la justice environnementale et des revendications des mouvements sociaux s'en réclamant, amenant à intégrer les notions de reconnaissance et de participation auparavant négligées.

\section{La justice environnementale comme juste reconnaissance et juste participation}

9 Les théories de justice environnementale mettent actuellement en lumière les injustices basées sur le déni de reconnaissance, entendu comme un "processus d'irrespect, insulte et dégradation dévaluant certains individus (...) au regard des autres " (Walker, 2009, p. 615, trad. pers.). A contrario la reconnaissance viserait à "préserver, respecter et permettre le plein épanouissement des identités individuelles et collectives » (Svampa, 2011, p. 116). Ces réflexions, fondées à la fois sur des avancées théoriques en justice sociale et sur des analyses empiriques, ont donné lieu à un renouvellement de la notion de justice environnementale. Elle est dès lors vue, sous l'égide de Schlosberg $(2004,2007)$ et Walker (2009) comme tridimensionnelle (suivant le cadre de Nancy Fraser considérant la reconnaissance, la redistribution et la parité de participation comme co-conditions de justice), ainsi que plurielle (pluralité des critères et principes définissant une procédure comme juste) (Martin et al., 2014). L'attention portée à la multi-dimensionnalité et à la pluralité de la justice ne doit toutefois pas être réduite à une tentative de la compartimenter. Il est important de garder à l'esprit que « les groupes et mouvements sociaux emploient souvent de multiples conceptions de la justice de manière simultanée, et acceptent à la fois l'ambiguïté et la pluralité qui accompagnent de tels discours hétérogènes »(Schlosberg, 2007, p. 5, trad. pers.).

10 C'est cette attention qui nous fait questionner la vision et les dimensions de justice qui sous-tendent le discours bolivien et plus spécifiquement celui de sa récente proposition à la COP 21 afin de mettre en lumière les différents registres de justification qui s'y rapportent et partant, les visions et les notions de justice auxquelles il se réfère, qui sont enchâssées dans un contexte bolivien dont nous rendrons ci-après succinctement compte.

\section{La proposition bolivienne à la COP 21: une volonté d'exportation d'un modèle de développement alternatif}

11 La contribution bolivienne à la COP 21 s'inscrit dans un contexte national ayant connu un bouleversement majeur depuis l'élection en 2005 d'Evo Morales (Webber, 2011). Celui-ci a refusé de poursuivre les politiques néolibérales menées par les gouvernements successifs de Paz Estensero et Paz Zamora et s'appuie, depuis 2009, sur une nouvelle Constitution censée assurer le « respect des droits indigènes et celui de l'environnement » (Perrier- 
Bruslé, 2012, p. 2). La Bolivie a dès lors mis en place des instruments légaux et des institutions climatiques et environnementales chargés de réguler de façon novatrice les diverses activités de mitigation et d'adaptation au changement climatique au niveau national, ainsi que leur financement.

12 À titre d'exemple, le récent "Mécanisme de mitigation et adaptation conjointe au changement climatique », illustre cette mutation politique. Il est défini comme "un processus de non-marchandisation de la forêt, basé sur la vie durable de la forêt» (Pacheco, 2012, s.p., trad. pers.) et géré par la nouvelle «Autorité Plurinationale de la Terre Mère $»^{4}$. Celle-ci vise à « articuler les efforts face au Changement Climatique et à la protection de la Terre Mère [Pachamama], avec l'intention de forger une vie digne pour les générations futures » selon son directeur Julio Gutiérrez (Fundación Natura Bolivia, 2015, s.p., trad. pers.). Ce mécanisme, mis en œuvre dès 2013, avait préalablement été reconnu comme un effort national légitime dans la lutte contre les émissions de GES lors de la conférence de Durban ${ }^{5}$, à la suite des pressions de la délégation bolivienne et de ses alliés. Dans le cadre de ce mécanisme, décliné notamment dans les Acuerdos Recíprocos por El Agua dans la province de Florida (Bétrisey \& Mager, 2015), les autorités boliviennes ne remettent en cause ni la notion de services environnementaux rendus par la forêt, ni le principe de leur compensation économique. Elles refusent toutefois -contrairement à l'initiative onusienne $\mathrm{REDD}^{+}{ }^{6}$ en faveur de la conservation de la forêt- de privilégier le service de séquestration du carbone sur d'autres services rendus par la forêt (tels que la biodiversité, les services dits culturels et d'approvisionnement en matières premières, etc.). De plus, le gouvernement bolivien s'oppose à ce que la compensation se fasse par le biais de transactions privées et ouvertes à l'international, privilégiant au contraire un mécanisme contrôlé par les autorités publiques suivant une logique de centralisationredistribution strictement nationale: «Nous ne nions pas le rôle du carbone, mais, pour nous, il s'agit d'une question minuscule. Les autres fonctions sont importantes. Nous ne nions pas l'existence de marchés justes pour la production forestière et que les produits forestiers jouent un rôle important dans les efforts des collectivités pour préserver leur forêt, mais nous ne voulons pas que les services fournis par la forêt soient soumis à des mécanismes de marché. Nous promouvons l'action collective et que des accord puissent se faire avec les communautés sans recourir à la commercialisation » (Pacheco, 2012, s.p., trad. pers.).

13 Le gouvernement national inscrit également le changement de la Bolivie dans un processus de "décolonisation", en dénonçant "les formes culturelles occidentales de l'État bolivien en tant qu'importations culturelles [qui] ne se rédui[raien]t pas au domaine de l'appareil étatique et de ses modalités d'administration, [mais] s'étend[raient] aussi aux relations interpersonnelles, aux modes d'interaction, à la culture matérielle et à l'imaginaire social " (Calvo \& Recondo, 2012, p. 184). Fer de lance de cette mutation désirée, la notion de "Vivir Bien", considérée comme symbole d'un développement " alternatif ». Relevant de différentes cosmologies indigènes andines, le «Vivir Bien » peut être défini comme « une vision holistique et intégrale » du développement, en " harmonie avec la Terre Mère» (Ministerio de Planificación del Desarrollo, 2015, p. 2, trad. pers.). Houtart (2011) estime que ce concept remplit une double fonction -de déconstruction et reconstruction- amenant, d'une part, à une critique proche des courants environnementalistes et féministes de la modernité, du progrès et du modèle de développement socio-économique occidental (Gudynas, 2011), et, d'autre part, à une proposition de reconstruction culturelle, sociale et politique, post-capitaliste (Houtart, 
2011, p. 7), visant à « construire une qualité de vie incluant autant les individus que la nature » (Gudynas, 2011, p. 2, trad. pers.). Différents auteurs questionnent toutefois la récupération politique de ce concept, lequel se transforme alors en une rhétorique de « la «redistribution du développement» (...) et conduit à promouvoir des politiques extractives ou de monocultures (en tant que sources de fonds destinés à être redistribués) utilisant le langage du Vivir Bien, sans même parler de transition » (Houtart, 2011: 9, trad. pers.).

14 Fort de ce que les autorités boliviennes présentent comme des succès nationaux en termes de réduction de la déforestation et de la pauvreté (Ministerio de Planificación del Desarrollo, 2015) et de ses diverses alliances internationales, le gouvernement bolivien, par l'intermédiaire de sa délégation aux Nations Unies, a proposé à la COP 21 un mécanisme de portée mondiale, censé distribuer de manière juste (Ministerio de Planificación del Desarrollo, 2015) les quotas d'émissions de GES. Cette proposition est la dernière d'une longue série de contestations émises dans des arènes diversifiées (conférences populaires, forums sociaux ${ }^{7}$, réunions gouvernementales), dans lesquelles il était fait référence aux notions d'équité et de justice climatique et environnementale (Evans, 2014).

15 En effet, le gouvernement d'Evo Morales et sa délégation aux Nations Unies (notamment au travers de son ambassadeur Pablo Solón) avaient déjà en 2009 remis publiquement en cause les mécanismes de distribution des quotas d'émissions de GES et des ressources financières destinées aux activités de mitigation et d'adaptation au changement climatique. Ils en appelaient alors, conjointement à d'autres délégations de pays du Sud et de mouvements sociaux, à ce que des notions telles que la «dette écologique et climatique » et les « responsabilités communes mais différenciées » (Pacheco, 2012, s.p., trad. pers.) soient reconnues, allant même jusqu'à parler de «réparation » ou de «remboursement» (Pablo Solón ${ }^{8}$, cité dans Martinez-Alier, 2011, p. 134). De plus, le gouvernement bolivien a mené dès 2009 une stratégie d'alliances avec des acteurs de la société civile, en particulier des mouvements sociaux indigènes et des organisations environnementales (Evans, 2014), mais aussi avec des délégations gouvernementales des pays «Non-Annexe I» dans le protocole de Kyoto (c'est-à-dire de pays en développement), ayant amené à la création d'un "groupe d'affinité » avec les pays du G-779 ${ }^{9}$ dont la Bolivie assume la présidence depuis 2013. Certains responsables du gouvernement bolivien en viennent à poser, sans le démontrer, que la Bolivie serait un acteur-clé des négociations internationales: «Depuis 2009, la Bolivie a cessé d'être un suiveur, pour développer sa propre position politique » (Orellana ${ }^{10}, 2012$, trad. pers.).

Les nouvelles pistes présentée par la Bolivie à la COP 21 pour transformer la gouvernance internationale du climat s'appuyaient sur 1) un indice de justice climatique, censé servir de base à un nouveau modèle de distribution des droits d'émissions de GES ; 2) l'institutionnalisation d'un tribunal compétent pour statuer du respect des critères proposés par la Bolivie dans l'attribution des droits d'émission et du respect des seuils définis. Ce faisant, le gouvernement bolivien entendait non seulement rendre la gouvernance globale du climat plus juste, mais également bannir le «modèle de civilisation consumériste, belliciste et mercantiliste du capitalisme [et proposer un nouvel] horizon culturel de civilisation alternatif au capitalisme " (Ministerio de Planificación del Desarrollo, 2015, p., 2, trad. pers.).

17 Cet indice se compose des paramètres suivants, mesurés au niveau des États:

- la responsabilité historique (mesurée par les émissions historique de GES depuis 1750), 
- l'empreinte écologique actuelle,

- la capacité de développement (mesurée conjointement et de manière non-pondérée par le PIB, le \% d'individus vivant au-dessous du seuil de 1,25 USD par jour et l'IDH),

- la capacité technologique (mesurée par l'Indice de performance compétitive de l'industrie élaboré par l'Organisation des Nations Unies pour le développement industriel (UNIDO) et le montant des dépenses en Recherche \& Développement comme pourcentage du PIB)

- et enfin la population.

En simulant l'adoption d'un tel indice pour répartir les droits d'émissions de GES, on arriverait au résultat suivant: les pays de l'«Annexe 1 » (développés) se verraient attribuer $11 \%$ des droits d'émissions, ce qui signifierait une baisse drastique des émissions actuelles, et les pays « Non-Annexe 1 » se verraient attribuer $89 \%$ du total des émissions de GES autorisées (Ministerio de Planificación del Desarrollo, 2015).

Il ne s'agit donc pas d'augmenter le total des émission de GES permises mais de les distribuer de manière différente, garantissant ainsi, selon la délégation bolivienne, le respect d'un «droit au développement » (Orellana, 2013, s.p., trad. pers.). Ce faisant, le gouvernement d'Evo Morales chercherait à assurer que « les pays du Sud ne soient pas les gardes forestiers des pays du Nord et du système capitaliste $»^{11}$. Enfin, comme évoqué précédemment, la délégation bolivienne proposait également, dans sa contribution à la COP 21, la constitution d'un tribunal climatique, chargé de faire respecter la règle de redistribution. Ce tribunal pourrait être amené à juger des actions en réparation et en dédommagement, dans le cas d'atteintes environnementales liées au changement climatique affectant les pays en développement et dont la responsabilité aurait été attribuée aux pays développés.

\section{La proposition bolivienne à la COP 21 au prisme des différentes notions de justice}

La proposition bolivienne faisait implicitement référence à la vision distributive de la justice environnementale, inspirée d'une interprétation rawlsienne de celle-ci. Rappelons qu'elle ambitionne la compensation des inégalités injustes, soit celles qui ne profitent pas aux plus démunis, par des mécanismes de redistribution.

21 La COP 21 permettait ainsi à la Bolivie de remettre en cause la gouvernance actuelle du climat, en affirmant que les pays du Sud -et en particulier la Bolivie- seraient victimes d'injustices dans la mesure où ce sont les pays développés qui ont émis la majorité des émissions de gaz à effet de serre dans l'atmosphère. Dans cette perspective, explicitement soutenue par le vice-président bolivien García Linera, il y a injustice car l'espace atmosphérique "polluable» sans incidence climatique majeure a été historiquement accaparé par les pays développés, restreignant aujourd'hui dans les pays actuellement en développement la possibilité d'utiliser des vecteurs énergétiques générateurs de GES pour accompagner leur croissance économique (p.e. le charbon, ayant l'avantage d'être relativement bon marché). Cette conception est en résonance avec la notion d'« accumulation par contamination " proposée par Martinez-Alier (2011, p. 134). Compte tenu de la pauvreté «endémique» en Bolivie (Pacheco, 2012, s.p., trad. pers.), le gouvernement considère qu'émettre des GES relève d'un droit au développement (Corz, 2015). Limiter le droit de produire des GES en Bolivie est donc vu comme une injustice -de plus- faite au détriment des plus vulnérables. Lutter contre cette injustice devrait alors, 
selon les autorités boliviennes, passer par la mise en place de mécanismes redistributifs inégalitaires, qui, s'ils favorisent les plus démunis, induiront plus de justice. Cette conception s'inscrit donc dans le champ idéologique des "réparations historiques » faisant références aux processus d'appropriation par dépossession qui auraient suivi le " péché originel » de la colonisation (Santos, 2011, p. 28) -marquée par une économie de traite ayant induit ce que Perrier-Bruslé (2008, p. 243) appelle le « syndrome de Potosí »et qui n'ont à ce jour pas amené à des réparations. C'est à ce titre qu'a été développé l'indice de justice climatique, censé guider une redistribution "juste» des droits d'émissions de GES.

Ce faisant, l'indice climatique ne s'oppose pas à la dimension redistributive qui prime au sein de la gouvernance climatique, mais critique implicitement, dans une perspective néo-marxiste à la Harvey, les critères de distribution actuels, qui seraient basés sur le «mérite » et la domination et non sur le besoin et la responsabilité. La proposition bolivienne s'aligne sur le discours de nombreux mouvements de promotion de la justice environnementale, souvent latino-américains, plaidant pour la prise en compte de la notion de dette écologique dans la constitution des mécanismes actuels de gouvernance internationale de l'environnement et du climat (Martinez-Alier, 2011). Toutefois, jusqu'à présent, ces "tentatives d'équilibrage entre le droits des pays en développement à se développer et le besoin de décarboner l'économie globale " (Newell \& Mulvaney, 2013, p. 136, trad. pers.) n'ont guère eu de succès au sein des négociations internationales sur le climat (Bond, 2012).

Cependant, au-delà de cette dimension redistributive, la proposition bolivienne fait aussi état de la nécessité de protéger la Terre Mère -soit le monde non-humain, reconnu comme « une communauté indivisible de tous les systèmes de vie et êtres vivants, interreliés, interdépendants et complémentaires qui partagent une destinée commune" (Ministerio de Planificación del Desarrollo, 2015, p. 2, trad. pers.)- des atteintes environnementales, en lui reconnaissant des droits. La Constitution bolivienne ${ }^{12}$ reconnaît d'ailleurs, dans un tournant juridique « bio-centré » (Gudynas, 2009) des droits à la Nature (Svampa, 2011). Ce discours entend ainsi dépasser une vision de la justice centrée sur la distribution juste (quel que soit le principe sur lequel l'équité de distribution est amenée à se baser), pour inclure les dimensions de reconnaissance et de participation du monde non-humain. Cela satisfait de jure aux conditions de justice écologique au sens de Schlosberg, définie comme une «justice entre les humains et le reste du monde naturel » (Schlosberg, 2007, vii, trad. pers.), tout en élargissant la vision rawlsienne de la justice, ne serait-ce que parce que l'on envisage que le monde nonhumain puisse être représenté devant un tribunal compétent. Le gouvernement bolivien estime par ailleurs que cela contribuera à la reconnaissance des populations indigènes à travers la reconnaissance de leurs "cosmovisions", lesquelles promeuvent justement l'harmonie et le respect du monde non-humain, même s'il convient de se rappeler que ces cosmovisions ont été influencées par le mode de pensée et de développement occidental (Zoomers, 2006) et de ne pas postuler de culture indigène essentialisée et radicalement autre.

Toutefois, la contribution bolivienne, à ce stade, ne précise pas comment cette reconnaissance et cette participation pourraient se concrétiser de facto. Certes le gouvernement d'Evo Morales a organisé à deux reprises (2010 et 2015) des sommets alternatifs, dénommés "conférences mondiales des peuples contre le changement climatique ", supposés favoriser la participation de la société civile, y compris les représentants des 
peuples indigènes. Mais ces évènements restent déconnectés des négociations internationales et plusieurs mouvements sociaux boliviens -souvent indigènes- ont affirmé s'être sentis exclus, du sommet de 2010 en tout cas (Aguirre \& Cooper, 2010). En l'état, l'indice de justice climatique se contente de contester les principes sous-jacents à la distribution des quotas nationaux d'émission de GES en proposant une distribution basée sur les besoins et les responsabilités, matérialisés dans les concepts de droit au développement et de dette climatique. De fait, cet indice ne traite aucunement des dimensions de reconnaissance, ni d'ailleurs de procédure et de participation. Si la vision multi-dimensionnelle de la justice sous-tend bien le discours de la proposition bolivienne (en cohérence avec le discours du gouvernement au niveau national), les pistes d'actions et outils proposés sont donc uniquement basés sur la redistribution (des droits d'émission) et la volonté d'assurer à la Bolivie sa part de GES -aussi «justifiée » soit-elle. Or, s'il n'est pas question d'invalider la dimension distributive de la justice, la concentration exclusive sur cette dimension passe «à côté de la question du cadrage préalable des valeurs qui constitue un mécanisme plus fondamental de production d'injustices » (Martin et al., 2013, p. 100, trad. pers.) et, ainsi, n'offre aucune " garantie de respect» (Martin et al. 2013, p. 124, trad. pers.) et de satisfaction des dimensions de reconnaissance et de participation constitutives de justice.

La notion de justice distributive mobilisée par la Bolivie a ainsi le défaut d'être essentiellement conséquentialiste, évaluant les résultats (une redistribution équitable) tout en mettant réflexivement de côté le caractère (in)juste des processus aboutissant à telle ou telle situation (équité de participation et de reconnaissance). De plus, accepter l'équation [droit au développement = droit d'émettre des GES] revient à admettre qu'il est impossible de se développer d'une autre façon, soit en cherchant à minimiser les émissions de GES. Ceci amène à considérer comme juste l'exploitation/contamination de la nature au profit du développement de la Bolivie, donc, paradoxalement, à ne pas rejeter le mode de développement environnementalement prédateur mais créateur de "capital produit et humain» (Banque Mondiale, 2006) des pays développés, préalablement dénoncé du fait de ses impacts négatifs sur la Terre Mère.

\section{Les ambiguïtés du « néo-développementisme » bolivien}

De fait, le gouvernement Morales promeut, malgré des références récurrentes à la nécessité de protéger la nature, une logique économique dite " néo-développementiste " $^{13}$ (Svampa, 2011, p. 110) tendant à encourager l'extractivisme -au sens d'un "modèle d'accumulation fondé sur la surexploitation des ressources naturelles » (op. cit., p. 105)-, sous couvert de la promesse d'une redistribution juste de la rente par la création et le soutien aux infrastructures publiques dans les domaines de la santé, de l'éducation et des transports. Svampa (2011) montre le parallèle entre cette logique et celle des avantages comparatifs de la doxa néolibérale, qu'une grande partie des gouvernements latinoaméricains actuels, à l'instar du gouvernement bolivien, critiquent pourtant.

Au-delà de l'exploitation des ressources naturelles, cette ambiguïté du discours officiel oscillant entre dénonciation d'un modèle de développement eurocentriste et néodéveloppementisme- a été révélée lorsque le gouvernement national a imposé la construction d'une route traversant le «Territoire Indigène et Parc Pational Isiboro- 
Secure » (TIPNIS), sans respecter le principe de consultation préalable des communautés indigènes locales, entraînant des protestations et des marches qui furent réprimées avec violence (Perrier-Bruslé, 2012). Cet épisode a permis d'illustrer le « regain de centralisme étatique » (op. cit.:, p. 35) ${ }^{14}$ du gouvernement bolivien, cherchant à maintenir son contrôle sur le territoire et mettant à mal l'idéal d'une Bolivie «autonomiste, plurinationale et communautaire » (op. cit., p. 33), pourtant inscrit dans la Constitution de 2009. Pour justifier cette politique, "Evo Morales s'appu[yait] sur l'axiome qui veut que la construction de route soit la garantie du développement et de la lutte contre la pauvreté » (op. cit., p. 30), les opposants à la route du TIPNIS étant vus comme des "empêcheurs" du développement, voire des ennemis de la Nation. Poupeau (2013) estime quant à lui que la rhétorique « du Vivir Bien et de la Pachamama, qui devrait être préservée de la frénésie capitaliste, peut même sembler jouer une fonction de «compensation» politique par rapport aux realpolitiks menées en matière d'hydrocarbures. Cette ambiguïté se retrouve au niveau de la Constitution politique de l'État approuvée par référendum en 2009, qui reste très formelle et peut paraître déconnectée des politiques publiques mises en œuvre au même moment» (op. cit., p. 112). Ces contradictions sont surtout visibles et analysées au niveau interne, préservant à l'étranger l'image de la Bolivie, encore souvent perçue comme un «laboratoire d'un monde meilleur pour les Indiens et la Nature » (Perrier-Bruslé, 2012, p. 22).

Certes le gouvernement bolivien assure que la souscription au modèle extractiviste n'est que temporaire, jusqu'à l'obtention d'un certain «niveau de développement»-au demeurant non spécifié- à partir duquel il serait possible de «remplacer les valeurs culturelles toujours plus individualistes, consuméristes et utilitaristes au sein de la société bolivienne » (Republic of Bolivia, 2002, cité dans Evans, 2014, p. 21, trad. pers.). Cela revient tout de même à être en contradiction avec la volonté exprimée par les autorités boliviennes de promouvoir l'équité de reconnaissance et de participation du monde non-humain. Les éléments considérés comme propres aux cosmologies indigènes et aux individus qui les revendiquent sont marginalisés, en contradiction avec le discours officiel prétendant: « construire et consolider un ordre mondial du Vivir Bien, défendant et promouvant les droits intégraux de nos peuples, prenant le chemin de l'harmonie avec la nature et du respect de la vie» (Ministerio de Planificación del Desarrollo, 2015, p. 6, trad. pers.). De plus, Laing (2012) a montré que le gouvernement bolivien ne renonçait pas aux " binarités de " civilisation/sauvagerie », et " modernité/tradition », en stigmatisant souvent dans ses communications «les «indiens" passéistes (indigènes des basses terres)» (op. cit., p. 1052, trad. pers.), malgré le discours postcolonial du gouvernement Morales, porté notamment par le vice-président García Linera.

En conséquence, si la contribution bolivienne suggère la reconnaissance juridique du monde non-humain par l'attribution de droits à la Terre Mère, elle ne se donne pas les moyens d'établir une justice véritablement écologique au sens de Schlosberg (2007), dans la mesure où les outils proposés tendent plutôt à justifier l'exploitation de la nature par le biais de sa transformation en capital produit et humain et non à établir une justice entre le monde humain et le monde non-humain.

\section{Conclusion}

30 La Bolivie affirmait faire pour la COP 21 des propositions qui amèneraient à plus de justice, tant par la redistribution en faveur des plus démunis que par la reconnaissance et 
la participation des peuples indigènes et du monde non-humain. Ces propositions restent toutefois limitées à une revendication de principe pour ce qui relève des dimensions participatives et de reconnaissance de la justice. En effet, le cadre réflexif proposé se limite à la dimension redistributive. Ce faisant, les propositions boliviennes ne contestent pas la conception actuelle de la justice environnementale, sous-jacente à la gouvernance climatique, mais se contentent de tenter d'en infléchir le résultat. De plus, les outils proposés, dont l'indice de justice climatique, justifient, par leur dimension pragmatique et conséquentialiste, le modèle de développement anthropocentré et environnementalement prédateur que le gouvernement bolivien condamne dans les arènes de discussion internationales (Poupeau, 2013).

31 La contribution bolivienne, proposant pourtant d'attribuer des droits au monde nonhumain, y compris celui d'être représenté devant un tribunal compétent, apparait de fait incapable de satisfaire aux conditions de justice écologique au sens de Schlosberg. Sans préjuger de la nécessité pour les pays "Non-Annexe I» de s'affirmer en contestant au sein de la gouvernance climatique les mécanismes d'appropriation et «l'échange écologique inégal » (Martinez-Alier, 2011, p. 139), nous constatons que la proposition bolivienne s'éloigne de l'alliance jugée prometteuse par Martinez-Alier (2011) entre justice environnementale et décroissance économique, de même qu'entre les mouvements socio-environnementaux qui les revendiquent. Ainsi, au lieu de «décoloniser les ressources naturelles des visions coloniales biaisées» (Ministerio de Planificación del Desarrollo, 2015, p. 7, trad. pers.), la contribution bolivienne et les dispositifs proposés par le gouvernement ont plutôt tendance à justifier et à prolonger le modèle de développement occidental "encastré dans une épistémologie coloniale " (Laing, 2012, p. 1051, trad. pers.). Svampa (2011) estime d'ailleurs que c'est la Bolivie qui «offre le panorama latino-américain le plus emblématique de l'illusion développementiste et le plus paradoxal » (op. cit., p. 112).

Certes, il est difficile, comme le montre Boaventura de Sousa Santos (cité dans Ziai, 2013) de mettre en place des modes de développement alternatifs au modèle de développement eurocentrique dominant, ce dernier se caractérisant par son habile décrédibilisation et invisibilisation de toute contestation. On peut toutefois constater que la position néodéveloppementiste extractiviste d'Evo Morales, réitérée lors de la COP 21 à Paris, fait l'objet de nombreuses critiques, y compris au sein de la classe politique bolivienne, à l'instar de Pablo Solón qui avait au demeurant écrit une lettre ouverte au président questionnant l'ambiguité de sa politique environnementale lors de l'affaire du TIPNIS (Solón, 2011). S'exprimant récemment dans le magazine L'Anticapitaliste Hebdo, Pablo Solón estime qu'au lieu de justice climatique, c'est à un processus de légalisation du « crime contre le climat, contre les migrants climatiques, contre les peuples indigènes des zones rurales et des pays insulaires" (Solón, 2015, s.p.) que participe aujourd'hui la Bolivie. Reste à savoir si les contestations qui se font jour dans la classe politique bolivienne pourront initier en Bolivie un récit post-extractiviste contribuant à concrétiser la promesse du " Vivir Bien » et de la justice écologique. 


\section{BIBLIOGRAPHY}

Aguirre J.C. \& Cooper, E.S., 2010. Evo Morales, Climate Change, and the Paradoxes of a SocialMovement Presidency. Latin American Perspectives, 37(4), p. 238-244.

Amin S., 1988. L'eurocentrisme: critique d'une idéologie. Paris, Anthropos.

Anthias P. \& Radcliffe S.A., 2013. The ethno-environmental fix and its limits: Indigenous land titling and the production of not-quite-neoliberal natures in Bolivia. Geoforum. http:// doi.org/10.1016/j.geoforum.2013.06.007

Banque Mondiale, 2006. Where is the wealth of nations? Measuring capital for the 21st century (Vol. 20). Washington, DC: The International Bank for Reconstruction and Development.

Barbière C., 2015. Contre le réchauffement climatique, la Bolivie appelle à détruire le capitalisme. http:// www.euractiv.fr/sections/climat-environnement/contre-le-rechauffement-climatique-labolivie-appelle-detruire-le

Bétrisey F. \& Mager C., 2015. Les paiements pour services environnementaux de la Fondation Natura Bolivia entre logiques réciprocitaires, redistributives et marchandes. Revue Française de Socio-Économie, 15(1), p. 39. http://doi.org/10.3917/rfse.015.0039

Blanchon D., Moreau S., \& Veyret Y., 2009. Comprendre et construire la justice environnementale. Annales de Géographie, 665-666(1), p. 35-60. http://doi.org/10.3917/ag.665.0035

Bond P., 2012. Politics of Climate Justice: Paralysis Above, Movement Below. Scottsville, South Africa, University of KwaZulu-Natal Press.

Calvo V. \& Recondo D., 2012. Chapitre 5/Bolivie : le gouvernement d'Evo Morales entre décolonisation de l'état et clientélisation politique. Académique, p. 175-199.

Corz C., 2015. García asegura que Bolivia no es culpable del cambio climático y defiende su derecho al progreso. La Razon Digital. La Paz, Bolivia. Consulté à http://obccd.org/2015/10/09/ garcia-asegura-que-bolivia-no-es-culpable-del-cambio-climatico-y-defiende-su-derecho-alprogreso/

Evans B. J., 2014. The plurinational State of Bolivia's Consideration of Ethics and Justice Issues in Formulating Climate Change Policies. In D. Brown \& P. Taylor (Eds.), Ethics and Climate Change: A study of national Commitments. Gland, IUCN, Vol. 86, p. 15-25.

Flitner M., 2009. « Nous sommes une poubelle... » : Échelles de reconnaissance et engagement de la société civile. Annales de géographie, 668(4), p. 397-413. http://doi.org/10.3917/ag.668.0397

Fundación Natura Bolivia, 2015. CONVENIO CON LA AUTORIDAD DE LA MADRE TIERRA. http:// www.naturabolivia.org/index.php?option=com_content\&view=article\&id=151\%3Aconvenio-conla-autoridad-de-la-madre-tierra\&catid=5\%3Anoticias\&Itemid=24\&lang=es

Gudynas E., 2009. La ecología política del giro biocéntrico en la nueva Constitución de Ecuador. Revista de Estudios Sociales (Bogotá), (32), p. 34-47.

Gudynas E., 2011. Buen Vivir: Germinando alternativas al desarrollo. América Latina En Movimiento, 462 , p. $1-20$.

Harvey D., 1996. Justice, nature and the geography of difference. Oxford: Blackwell. 
Hir A.G., 2015. A la COP 21, un compromis guidé par la « justice climatique ». Le Monde.fr. http:// www.lemonde.fr/cop21/article/2015/12/14/cop21-un-compromis-guide-par-la-justiceclimatique_4831575_4527432.html

Houtart F., 2011. El concepto del Sumak Kawsay (Buen Vivir) y su correspondencia con el bien común de la humanidad. Journal Ecuador Debate, 84. http://www.dhl.hegoa.ehu.es/ ficheros/0000/0738/15._El_concepto_de_sumak_kawsai.pdf

Joshi D., 2015. Like water for justice. Geoforum, 61, p. 111-121. http://doi.org/10.1016/ j.geoforum.2015.02.020

Laing A. F., 2012. Beyond the Zeitgeist of "Post-neoliberal" Theory in Latin America: The Politics of Anti-colonial Struggles in Bolivia. Antipode, 44(4), p. 1051-1054. http://doi.org/10.1111/ j.1467-8330.2012.01010.x

Martin A., 2013. Global environmental in/justice, in practice: introduction. The Geographical Journal, 179(2), p. 98-104. http://doi.org/10.1111/geoj.12021

Martin A., Gross-Camp N., Kebede B., McGuire S., \& Munyarukaza, J., 2014. Whose environmental justice? Exploring local and global perspectives in a payments for ecosystem services scheme in Rwanda. Geoforum, 54, p. 167-177. http://doi.org/10.1016/j.geoforum.2013.02.006

Martin A., McGuire S., \& Sullivan S. (2013). Global environmental justice and biodiversity conservation. The Geographical Journal, 179(2), p. 122-131. http://doi.org/10.1111/geoj.12018

Martinez-Alier, J., 2011. Justice environnementale et décroissance économique: l'alliance de deux mouvements. Ecologie \& Politique, 41(1), p. 125. http://doi.org/10.3917/ecopo.041.0125

Martínez-Alier J., Pascual U., Vivien F.-D., \& Zaccai E., 2010. Sustainable de-growth: Mapping the context, criticisms and future prospects of an emergent paradigm. Ecological Economics, 69(9), p. 1741-1747. http://doi.org/10.1016/j.ecolecon.2010.04.017

Ministerio de Planificación del Desarrollo, 2015. Contribucion prevista determinada nacionalmente del estado Plurinacional de Bolivia. La Paz, Bolivia: Estado plurinacional de Bolivia. http:// www.planificacion.gob.bo/sites/folders/1.BOLIVIA\%20CONTRIBUCI\%C3\%93N\%20\%28doc\%29.pdf Newell P. \& Mulvaney D., 2013. The political economy of the "just transition." The Geographical Journal, 179(2), p. 132-140. http://doi.org/10.1111/geoj.12008

Orellana R., 2012. Posicion Boliviana en Cambio Climatico. Presented at the Taller Nacional Posicion Internacional Boliviana en Cambio Climatico y Ley Marco de la Madre Tierra y Desarollo Integral para Vivir Bien, La Paz, Bolivia.

Orellana R., 2013. Lecture and Round Table on the Bolivian Strategy of Climate Change Adaptation and Mitigation. Présenté à la Conférence de l'Institut des Sciences de l'Environnement (UNIGE), Université de Genève, Suisse.

Pacheco D., 2012. Presentacion Mecanismo Cojunto de Adaptacion y Mitigacion para Manejo integral de los Bosque y la Madre Tierra. Présenté au Taller Nacional Posicion Internacional Boliviana en Cambio Climatico, La Paz, Bolivia.

Page E.A., 2011. Climatic Justice and the Fair Distribution of Atmospheric Burdens: A Conjunctive Account. The Monist, 94(3), p. 412-432.

Perreault T., 2014. What kind of governance for what kind of equity? Towards a theorization of justice in water governance. Water International, 39(2), p. 233-245. http:// doi.org/10.1080/02508060.2014.886843

Perrier-Bruslé, L., 2008. Le gaz bolivien. Outre-Terre, 18(1), p. 235-251. 
Perrier-Bruslé L., 2012. Le conflit du Tipnis et la Bolivie d'Evo Morales face à ses contradictions : analyse d'un conflit socio-environnemental. EchoGéo [En ligne], Sur le Vif, mis en ligne le 26 janvier 2012. http://echogeo.revues.org/12972 ; DOI : 10.4000/echogeo.12972

Policar A., 2006. La justice sociale : Les enjeux du pluralisme. Paris, Armand Colin.

Poupeau F., 2013. La Bolivie entre Pachamama et modèle extractiviste. Ecologie \& politique, 46(1), p. 109-119. http://doi.org/10.3917/ecopo.046.0109

Republic of Bolivia, 2002. National Climate Change Program: General bases for the application of the bolivian national implementation strategy of the United Nationa Framework Convention on Climate Change (National Climate Change Program). La Paz, Bolivian Ministry of Sustainable Development and Planning.

Robbins P., 2011. Political ecology: A critical introduction (Vol. 16). New York, John Wiley \& Sons. Santos B. de S., 2011. Épistémologies du Sud. Etudes Rurales, 187, p. 21-49.

Schlosberg D., 2004. Reconceiving Environmental Justice: Global Movements And Political Theories. Environmental Politics, 13(3), p. 517-540. http://doi.org/10.1080/0964401042000229025

Schlosberg D., 2007. Defining Environmental Justice: Theories, Movements, and Nature. Oxford: Oxford University Press.

Schroeder R., Martin K. S., Wilson B., \& Sen D., 2008. Third World Environmental Justice. Society \& Natural Resources, 21(7), p. 547-555. http://doi.org/10.1080/08941920802100721

Sen A., 2002. Rationality and Freedom. Harvard University Press.

Sikor T., Martin A., Fisher J. \& He J., 2014. Toward an Empirical Analysis of Justice in Ecosystem Governance. Conservation Letters, 7(6), p. 524-532. http://doi.org/10.1111/conl.12142

Solón P., 2011. Letter from Pablo Solón on the TIPNIS Highway Controversy. http:// www.boliviamundo.net/letter-from-pablo-Solón-on-the-tipnis-highway-controversy/ Solón, P., 2015. Un accord pour brûler la planète. L'Anticapitalste Hebdo, (310). http:// www.pressegauche.org/spip.php?page=imprimer\&id_article=24111

Svampa M., 2011. Néo-« développementisme » extractiviste, gouvernements et mouvements sociaux en Amérique latine. Problèmes d'Amérique latine, 81(3), p. 101-127.

UNFCCC, 2012. Report of the Conference of the Parties on its seventeenth session, held in Durban: Part Two: Action taken by the Conference of the Parties at its seventeenth session (No. FCCC/CP/2011/9/Add.1). United Nations.

Vanderheiden S. \& Sisson M., 2010. Ethically Responsible Leisure? Promoting Social and Environmental Justice Through Ecotourism. Environmental Philosophy, 7(2), p. 33-47.

Walker G., 2009. Beyond Distribution and Proximity: Exploring the Multiple Spatialities of Environmental Justice. Antipode, 41(4), p. 614-636. http://doi.org/10.1111/ j.1467-8330.2009.00691.x

Webber J., 2011. From Rebellion to Reform in Bolivia: Class Struggle, Indigenous Liberation, and the Politics of Evo Morales. Chicago, Haymarket Books.

Young, I., 1990. Justice and the Politics of Difference. Princeton, Princeton University Press.

Ziai A., 2013. Boaventura de Sousa Santos: Interview with Boaventura de Sousa Santos. Development and Change, 44(3), p. 727-738. http://doi.org/10.1111/dech.12026 
Zoomers, A., 2006. Pro-Indigenous Reforms in Bolivia: Is there an Andean Way to Escape Poverty? Development and Change, 37(5), p. 1023-1046. http://doi.org/10.1111/j.1467-7660.2006.00511.x

\section{NOTES}

1. http://www.planificacion.gob.bo/sites/folders/1.BOLIVIA\%20CONTRIBUCI\%C3\%93N\%20\% 28doc\%29.pdf

2. La chrématistique est la science de l'enrichissement.

3. http://unfccc.int/resource/docs/2015/cop21/fre/lo9f.pdf

4. Elle-même dépendante du Ministère de l'Environnement.

5. L'article 66 de la Conférence des Parties $n^{\circ} 17$ de Durban, qui considère l'approche basée sur le marché comme appropriée, a en effet été complété par l'article 67 qui reconnait que des "nonmarket-based approaches, such as joint mitigation and adaptation approaches for the integral and sustainable management of forests as a non-market alternative that supports and strengthens governance, (...) could be developed » (UNFCCC, 2012, p. 15).

6. http://www.un-redd.org/aboutredd

7. Notamment la Conferencia Mundial de los Pueblos sobre el Cambio Climático y los Derechos de la Madre Tierra à Cochabamba, organisée en 2013 par le gouvernement bolivien et qui offrit à ce dernier une exposition médiatique nationale et internationale importante.

8. Ambassadeur de la Bolivie aux Nations Unies de février 2009 à la fin juin 2011, il est actuellement directeur exécutif de l'organisation altermondialiste Focus on the Global South.

9. Les membres de ce groupe ont par exemple validé la proposition bolivienne d'un mécanisme non-marchand d'adaptation au changement climatique et de mitigation, à l'exception du Brésil (Orellana, 2012).

10. Ancien responsable de la délégation bolivienne à l'UNFCCC et aujourd'hui ministre de la planification du développement.

11. https://www.youtube.com/watch?v=VxzjeBdLIu0, trad. pers.

12. Il en va de même en Equateur.

13. Dans la mesure où elle s'éloigne de la logique développementiste moderniste des années 1980, qui promouvait, à l'instar de la CEPAL, l'industrialisation par substitution aux importations et l'éloignement d'un extractivisme primaire.

14. Perrier-Bruslé (2012) va jusqu'à parler de « jacobinisme » (op. cit., p. 33).

\section{ABSTRACTS}

The contribution presented in December 2015 at the Climate Conference (COP 21) in Paris by Bolivian officials enjoyed a certain media coverage. It proposed lines of action, allowing both to achieve a more "just" climate governance and to build a new "civilization horizon" breaking with the consumerist, extractive and capitalist model of development. This article aims to question, through the prism of the different dimensions of justice being mobilized (redistribution, recognition and participation), the notions of justice on which the Bolivian contribution is based. It identifies two levels: that of discourse, and that of the tools offered. We show that favouring a 
redistributive conception of justice, paradoxically the Bolivian contribution ultimately tends not to exceed the development model to which it objected.

La contribution présentée lors de la Conférence sur le climat (COP 21) en décembre 2015 à Paris par les représentants boliviens a joui d'un certain écho médiatique. Elle entendait proposer des pistes d'action à double dividende, permettant à la fois d'aboutir à une gouvernance climatique plus « juste» et de construire un nouvel « horizon de civilisation » rompant avec le modèle de développement consumériste, extractiviste et capitaliste. Cet article a pour objectif de questionner, au prisme des différentes dimensions de la justice actuellement mobilisées (redistribution, reconnaissance et participation), les notions de justice sur lesquelles se fonde la contribution bolivienne, en différenciant deux niveaux: celui du discours et celui des outils proposés. Nous montrons qu'en privilégiant une conception redistributive de la justice, la contribution bolivienne tend paradoxalement in fine à ne pas pouvoir dépasser le «modèle de civilisation » et de développement qu'elle dénonçait.

\section{INDEX}

Mots-clés: justice climatique, justice environnementale, Bolivie, COP 21, Rawls

Keywords: Climatic justice, Environmental justice, Bolivia, COP 21, Rawls, Vivir Bien

\section{AUTHORS}

\section{FLORENCE BÉTRISEY}

Florence Bétrisey, florence.betrisey@unil.ch, est Collaboratrice de recherche à l'Institut de Géographie et Durabilité, Université de Lausanne. Elle a publié récemment :

- Bétrisey F., 2015. The conditions for the reproduction of the SAGUAPAC water cooperative in the city of Santa Cruz de la Sierra, Bolivia: discourse analysis. Articulo - Journal of Urban Research, (Special issue 7). http://doi.org/10.4000/articulo.2764

- Bétrisey F. \& Mager, C., 2014. Small Farmers in Florida Province, Bolivia: Reciprocity in Practice. Mountain Research and Development, 34(4), p. 369-374. http://doi.org/10.1659/MRD-JOURNAL-

D-14-00013.1

\section{CHRISTOPHE MAGER}

Christophe Mager, christophe.mager@unil.ch, est Maître d'Enseignement et de Recherche à l'Institut de Géographie et Durabilité, Université de Lausanne. 